\title{
Advances in Solar Energy
}

An Annual Review of

Research and Development

Volume 2 


\title{
Editorial Board
}

\section{Co-Editors-in-Chief}

Karl W. Böer

University of Delaware and SES, Inc., Newark, Delaware

John A. Duffie

University of Wisconsin at Madison, Madison, Wisconsin

\author{
Associate Editors \\ Douglas J. Balcomb \\ Los Alamos National Laboratory, Los Alamos, New Mexico \\ Brian Brinkworth \\ University College, Cardiff, United Kingdom \\ Harry Bungay \\ Rensselaer Polytechnic Institute, Troy, New York \\ Kinsell Coulson \\ Mauna Loa Observatory, Hilo, Hawaii \\ Baruch Givoni \\ Ben Gurion University, Beersheba, Israel \\ Roger van Overstraeten \\ Katholieke Universiteit Leuven, Leuven, Belgium \\ Morton Prince \\ Department of Energy, Washington, D.C. \\ Michael Seibert \\ Solar Energy Research Institute, Golden, Colorado \\ Horst Selzer \\ Erno-Raumfahrttechnik GmbH, Bremen, Federal Republic of Germany \\ Bernard Serafin \\ University of Arizona, Tucson, Arizona \\ Steven Szokolay \\ University of Queensland, Queensland, Australia \\ Irwin Vas \\ Flowind Corporation, Kent, Washington \\ Donald Watson \\ Branford, Connecticut \\ John I. Yellott \\ John Yellott Engineering Associates Inc., Phoenix, Arizona
}

\footnotetext{
A Continuation Order Plan is available for this series. A continuation order will bring delivery of each new volume immediately upon publication. Volumes are billed only upon actual shipment. For further information please contact the publisher.
} 


\section{Advances in Solar Energy \\ An Annual Review of Research and Development}

\section{Volume 2}

\section{Edited by}

\section{Karl W. Böer}

University of Delaware and SES, Inc.

Newark, Delaware

and

John A. Duffie

University of Wisconsin at Madison

Madison, Wisconsin

AMERICAN SOLAR ENERGY SOCIETY, INC.

Boulder, Colorado - Newark, Delaware

and

\section{PLENUM PRESS}

New York • London 
DOI 10.1007/978-1-4613-9951-3

(c) 1985 Plenum Press, New York

Softcover reprint of the hardcover 1st edition 1985

A Division of Plenum Publishing Corporation

233 Spring Street, New York, N.Y. 10013

All rights reserved

No part of this book may be reproduced, stored in a retrieval system, or transmitted in any form or by any means, electronic, mechanical, photocopying, microfilming, recording, or otherwise, without written permission from the Publisher 


\section{FOREWORD}

A number of significant changes have occurred in Advances in Solar Energy since Volume 1 appeared in 1982. The delays in publication of the second volume are the result of reorganization of the American Solar Energy Society, and the negotiation of a new publishing arrangement. Beginning with this volume, Advances is now published jointly by the Society and Plenum Press. The Editorial Board has been enlarged to be more representative of the different fields of solar energy conversion.

Production of Advances is being expedited through the use of modern word processing equipment and the $T_{\mathrm{E}} \mathrm{X}$ typesetting-editing program. We have gone to a single-column format to ease the problems of presenting long equations, and we expect that the user of the volume will find it easy to read. The use of $T_{\mathrm{E}} \mathrm{X}$ will make last minute updates possible. The external appearance of the volume matches that of Volume 1.

We expect that future volumes of this annual will be proceeding on schedule. We invite comments from users and correspondence from prospective authors of critical reviews.

Karl W. Böer

John A. Duffie 


\section{CONTENTS}

CHAPTER 1 The Measurement of Solar Radiation

Ronald Stewart, Daniel W. Spencer and Richard Perez

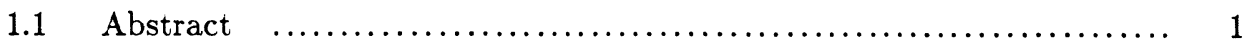

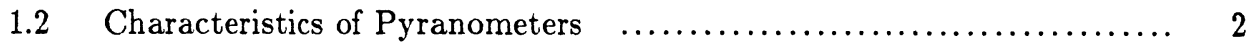

1.3 General Features of a Pyranometer $\quad \ldots \ldots \ldots \ldots \ldots \ldots \ldots \ldots \ldots \ldots . \ldots \ldots$

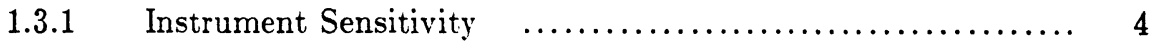

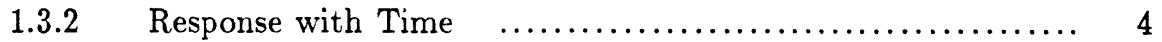

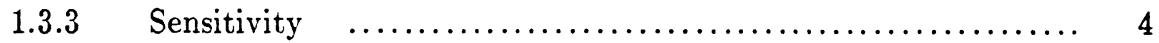

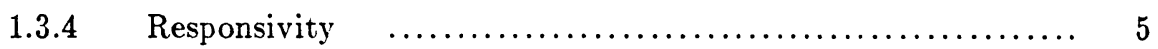

1.3.5 Temperature Coefficient of Sensitivity $\quad \ldots \ldots \ldots \ldots \ldots \ldots \ldots . \ldots$

1.3.6 Thermal Transient Response $\ldots . \ldots \ldots \ldots \ldots \ldots \ldots \ldots \ldots \ldots$

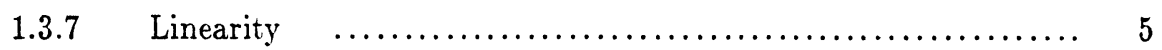

1.3.8 Angular Dependence of Sensitivity $\ldots \ldots \ldots \ldots \ldots \ldots \ldots \ldots$

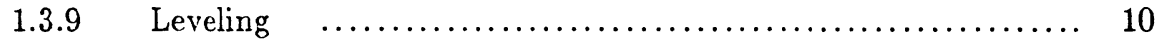

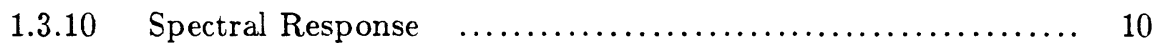

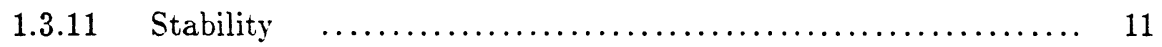

1.3.12 Pyranometer Sensitivity Function $\ldots \ldots \ldots \ldots \ldots \ldots \ldots \ldots . \ldots \ldots$

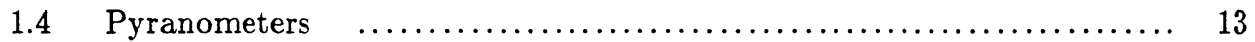

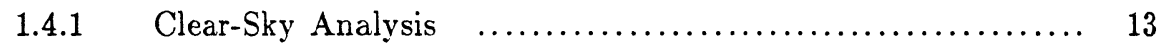

1.4.2 Installation of the Instrument $\ldots \ldots \ldots \ldots \ldots \ldots \ldots \ldots \ldots . \ldots \ldots$

1.4.3 Thermopile Construction $\quad \ldots \ldots \ldots \ldots \ldots \ldots \ldots \ldots \ldots \ldots, 16$

1.4.4 Moisture $\quad \ldots \ldots \ldots \ldots \ldots \ldots \ldots \ldots \ldots \ldots \ldots \ldots \ldots \ldots \ldots . \ldots \ldots$ 
1.4.5 Deposition $\quad \ldots \ldots \ldots \ldots \ldots \ldots \ldots \ldots \ldots \ldots \ldots \ldots \ldots \ldots \ldots, 16$

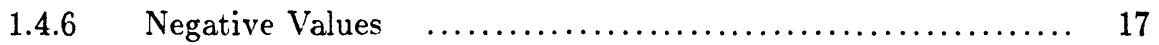

1.4.7 Readings Which Exceed the Values for Extraterrestrial Insola-

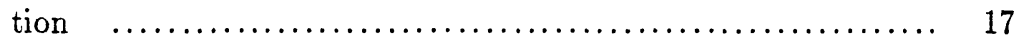

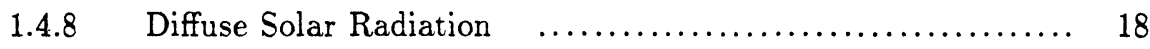

1.4.9 Shadowband Orientation $\quad \ldots \ldots \ldots \ldots \ldots \ldots \ldots \ldots \ldots \ldots \ldots . \ldots \ldots$

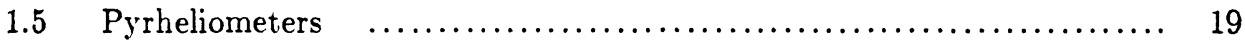

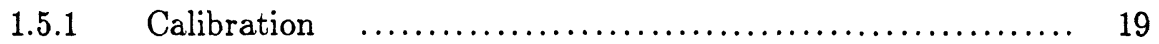

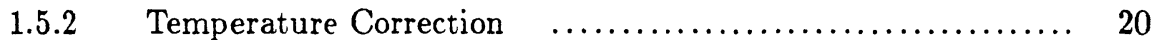

1.5.3 Spectral Measurements Using Broadband Filters $\ldots . \ldots \ldots . .20$

1.5.4 Time Constant of the Pyrheliometer $\ldots \ldots \ldots \ldots \ldots \ldots \ldots . . \ldots 21$

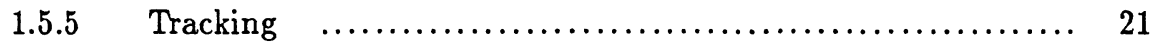

1.5.6 Importance of the Solar Aureole $\ldots \ldots \ldots \ldots \ldots \ldots \ldots \ldots . \ldots . \ldots 24$

1.5.7 Pyrheliometer Tracker Wiring $\ldots \ldots \ldots \ldots \ldots \ldots \ldots \ldots \ldots . . \ldots \ldots$

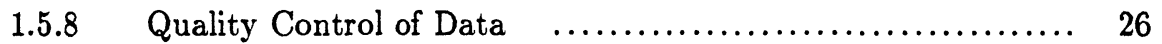

1.5.9 Solar Radiation Instruments $\ldots \ldots \ldots \ldots \ldots \ldots \ldots \ldots \ldots . \ldots \ldots$

1.5.9.1 The Pyrheliometers $\ldots \ldots \ldots \ldots \ldots \ldots \ldots \ldots . \ldots 28$

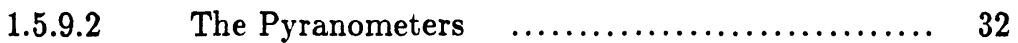

1.5.9.3 Duration of Sunshine Instruments $\ldots \ldots \ldots \ldots . . .38$

Appendix 1.1 Historical Perspectives $\ldots \ldots \ldots \ldots \ldots \ldots \ldots \ldots \ldots \ldots \ldots \ldots, 41$

1.1.1 Historical Perspectives-From Sundials to Satellites $\ldots . . . .41$

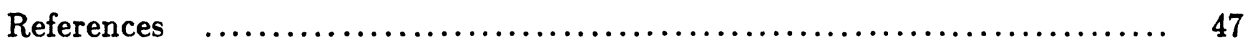

\section{CHAPTER 2 Environmental Requirements for Anaerobic Diges- tion of Biomass}

\section{R. E. Speece}

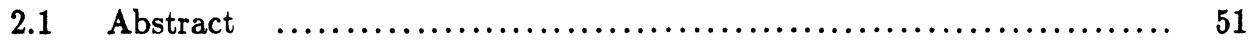

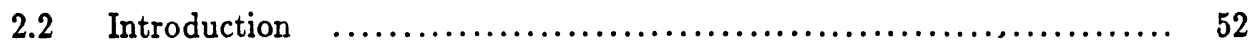

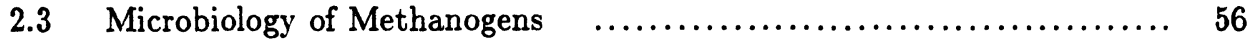

2.3.1 Interspecies $H_{2}$ Transfer $\quad \ldots \ldots \ldots \ldots \ldots \ldots \ldots \ldots \ldots \ldots \ldots, 58$

2.3.2 Methanogenic Reactions $\ldots \ldots \ldots \ldots \ldots \ldots \ldots \ldots \ldots \ldots \ldots . \ldots \ldots$

2.3.3 Characteristics of Methanogenic Species in Pure Culture . . 59 


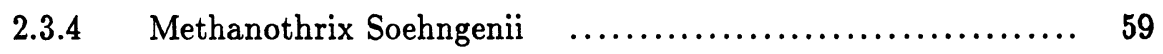

2.3.5 Biochemical Mechanisms and Pathways $\ldots . \ldots \ldots \ldots \ldots \ldots . . . \ldots$

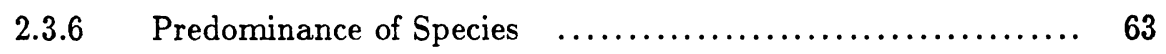

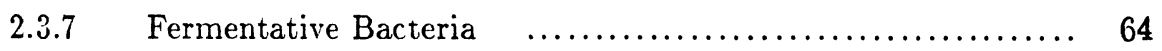

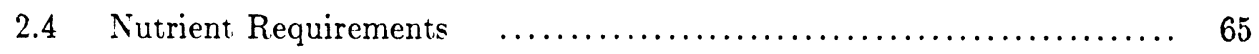

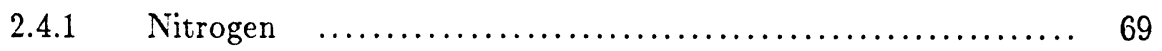

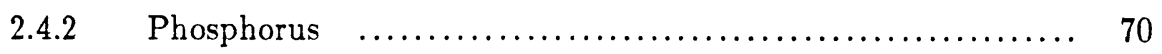

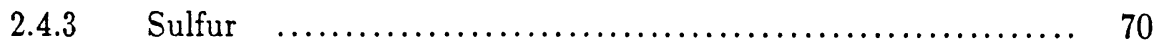

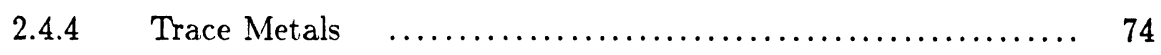

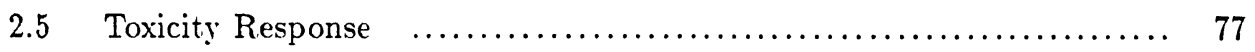

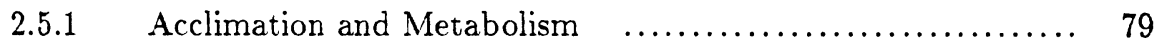

2.5.2 Microbial Kinetics and Toxicity $\ldots \ldots \ldots \ldots \ldots \ldots \ldots \ldots . \ldots . \ldots . \ldots$

2.5.3 Toxicity in Anaerobic Digestion Studies $\ldots \ldots \ldots \ldots \ldots \ldots . . .64$

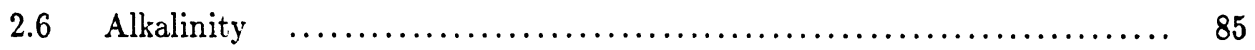

2.6.1 Alkalinity Concentration Required $\ldots \ldots \ldots \ldots \ldots \ldots \ldots . \ldots$

2.6.2 Choice of Purchased Alkalinity $\ldots \ldots \ldots \ldots \ldots \ldots \ldots \ldots . \ldots . \ldots . \ldots 1$

2.7 Modeling of the Anaerobic Digestion Process $\ldots \ldots \ldots \ldots \ldots \ldots \ldots \ldots . \ldots 8$

2.7.1 Cellular Synthesis of Organic Substrate $\ldots \ldots \ldots \ldots \ldots \ldots \ldots . . .68$

2.7.2 Rate-Limiting Step in Anaerobic Digestion $\ldots \ldots \ldots \ldots \ldots . . .69$

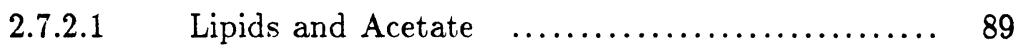

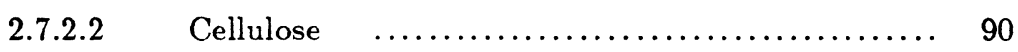

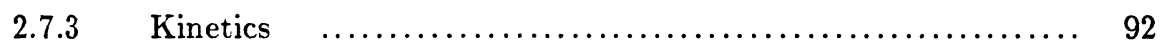

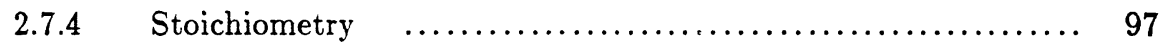

2.7.5 Calculations of Methane Production $\ldots \ldots \ldots \ldots \ldots \ldots \ldots . . \ldots 8$

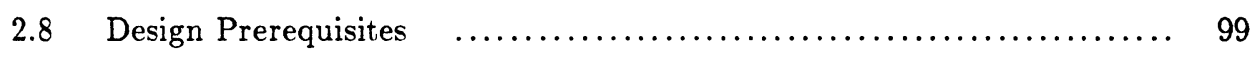

2.8.1 Solids Concentration in Feed Sludge $\ldots \ldots \ldots \ldots \ldots \ldots \ldots . \ldots 100$

2.8.2 Batch versus Continuous Feeding $\quad \ldots \ldots \ldots \ldots \ldots \ldots \ldots \ldots \ldots$

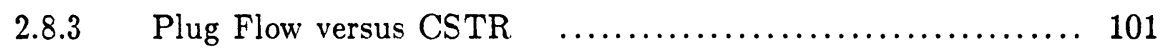

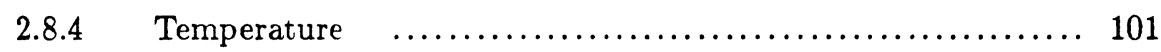

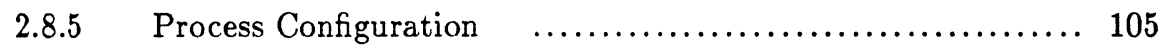

2.8.6 Soluble versus Sludge Feedstocks $\ldots \ldots \ldots \ldots \ldots \ldots \ldots \ldots \ldots$

2.8.7 Industrial Wastewaters Treated by Methane Fermentation . 106 


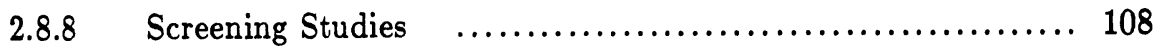

2.8.9 Scale-Up Factors Affecting Performance $\ldots . \ldots \ldots \ldots \ldots \ldots . . \ldots 109$

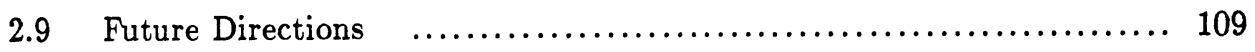

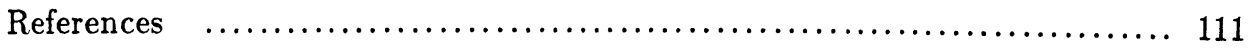

CHAPTER 3 Principles and Technology of Biomass Gasification

\section{Thomas B. Reed}

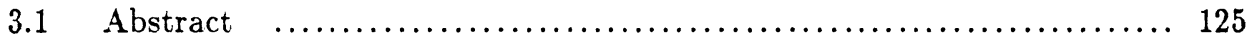

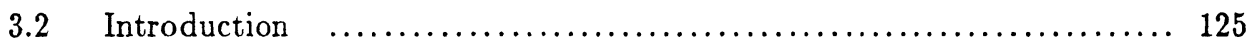

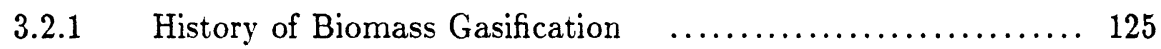

3.2.2 Major References on Biomass Gasification $\ldots \ldots \ldots \ldots \ldots \ldots . \ldots 127$

3.2.3 Gasification Research Centers $\quad \ldots \ldots \ldots \ldots \ldots \ldots \ldots \ldots \ldots \ldots \ldots \ldots \ldots$

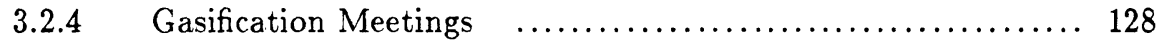

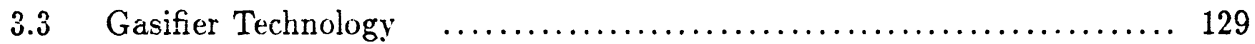

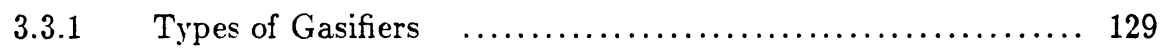

3.3.1.1 Charcoal Gasifiers $\quad$.......................... 129

3.3.1.2 Updraft Gasifiers $\quad \ldots \ldots \ldots \ldots \ldots \ldots \ldots \ldots \ldots . \ldots 129$

3.3.1.3 Downdraft Gasifiers $\quad \ldots \ldots \ldots \ldots \ldots \ldots \ldots \ldots \ldots . \ldots 130$

3.3.1.4 Fluid:zed Bed Gasifiers $\quad \ldots \ldots \ldots \ldots \ldots \ldots \ldots \ldots, 132$

3.3.1.5 Suspension Gasifiers $\quad \ldots \ldots \ldots \ldots \ldots \ldots \ldots \ldots \ldots \ldots$

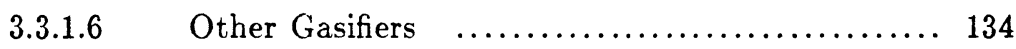

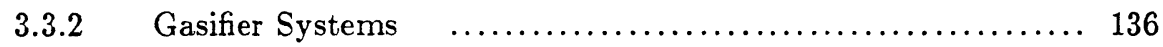

3.3.3 Gasifier System Specifications $\quad \ldots \ldots \ldots \ldots \ldots \ldots \ldots \ldots \ldots \ldots \ldots \ldots \ldots$

3.3.4 Producer Gas Use and Gas Conditioning f............. 138

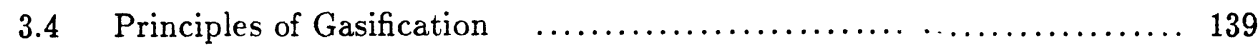

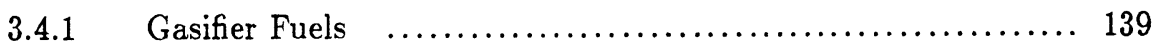

3.4.2 Biomass versus Coal Gasification $\quad \ldots \ldots \ldots \ldots \ldots \ldots \ldots \ldots . \ldots \ldots$

3.4.3 Chemistry of Biomass Gasification $\quad \ldots \ldots \ldots \ldots \ldots \ldots \ldots \ldots \ldots .143$

3.4.4 Global Thermodynamics of Biomass Gasification .......... 144

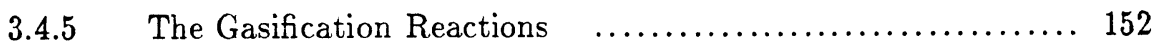

3.4.5.1 Solid and Gas Pyrolysis $\quad \ldots \ldots \ldots \ldots \ldots \ldots \ldots . \ldots 153$ 
3.4.5.2 Kinetics of Solid Pyrolysis Reactions ......... 155

3.4.5.3 Kinetics of Biomass Vapor Pyrolysis Reactions . 159

3.4.5.4 Mechanisms of Charcoal Gasification ......... 159

3.4.5.5 Kinetics of Charcoal Gasification $\ldots . \ldots \ldots \ldots . .160$

3.4.6 Modeling of Gasifier Operation $\quad \ldots . \ldots \ldots \ldots \ldots \ldots \ldots \ldots . \ldots . \ldots 2$

3.4.6.1 Updraft Gasifier Modeling $\quad \ldots \ldots \ldots \ldots \ldots \ldots \ldots . . \ldots 164$

3.4.6.2 Downdraft Gasifier Modeling $\ldots \ldots \ldots \ldots \ldots \ldots . \ldots 166$

3.4.6.3 Fluidized Bed Gasifier Modeling $\quad \ldots \ldots \ldots \ldots \ldots . . .167$

3.4.6.4 Fast Pyrolysis Modeling $\quad \ldots \ldots \ldots \ldots \ldots \ldots \ldots . \ldots 168$

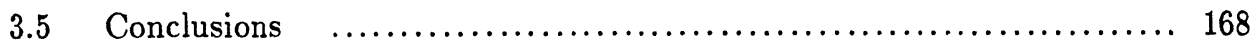

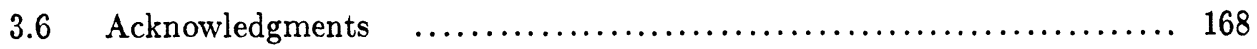

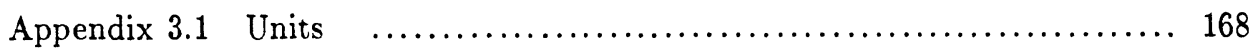

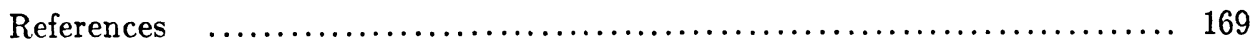

CHAPTER 4 Biomass Pyrolysis: A Review of the Literature Part 2-Lignocellulose Pyrolysis

Michael Jerry Antal, Jr.

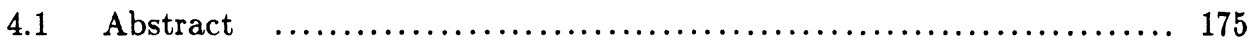

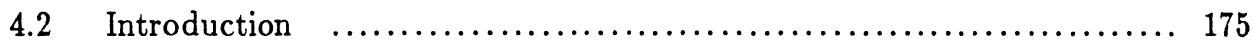

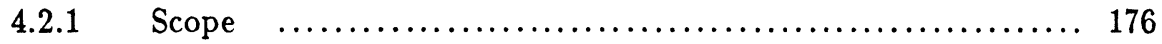

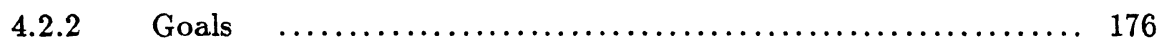

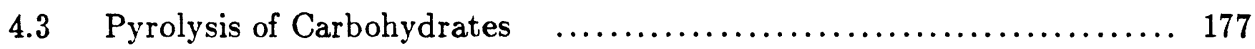

4.3.1 Low Temperature Phenomena $\ldots \ldots \ldots \ldots \ldots \ldots \ldots \ldots \ldots \ldots \ldots \ldots \ldots$

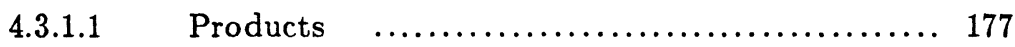

4.3.1.2 Mechanisms and Kinetics $\quad \ldots \ldots \ldots \ldots \ldots \ldots \ldots . \ldots 178$

4.3.2 Moderate Temperature Phenomena $\ldots . \ldots \ldots \ldots \ldots \ldots \ldots . \ldots 178$

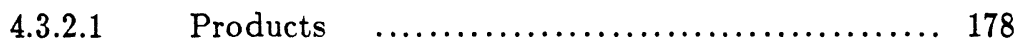

4.3.2.2 Mechanisms and Kinetics $\ldots \ldots \ldots \ldots \ldots \ldots \ldots . \ldots 179$

4.3.3 High Temperature Phenomena $\quad \ldots \ldots \ldots \ldots \ldots \ldots \ldots \ldots \ldots . \ldots \ldots$

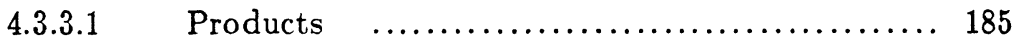

4.3.3.2 Mechanisms and Kinetics $\quad \ldots \ldots \ldots \ldots \ldots \ldots \ldots . \ldots 185$

4.3.4 Effects of Various Parameters $\quad$....................... 190 


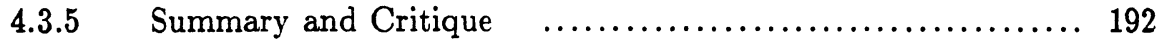

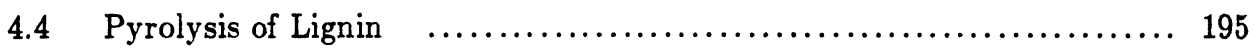

4.4.1 Low Temperature Phenomena $\ldots . \ldots \ldots \ldots \ldots \ldots \ldots \ldots . \ldots . \ldots$

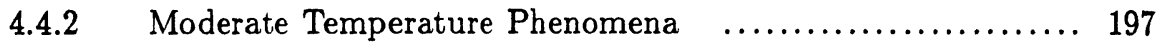

4.4.2.1 Products $\quad \ldots \ldots \ldots \ldots \ldots \ldots \ldots \ldots \ldots \ldots \ldots \ldots \ldots . \ldots \ldots$

4.4.2.2 Mechanisms and Kinetics $\ldots \ldots \ldots \ldots \ldots \ldots \ldots, 198$

4.4.3 High Temperature Phenomena $\ldots \ldots \ldots \ldots \ldots \ldots \ldots \ldots . \ldots . \ldots 206$

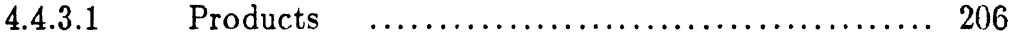

4.4.3.2 Mechanisms and Kinetics .................. 207

4.4.4 Effects of Various Parameters $\quad \ldots \ldots \ldots \ldots \ldots \ldots \ldots \ldots \ldots . \ldots . \ldots . \ldots 212$

4.4.4.1 Heating Rate $\ldots \ldots \ldots \ldots \ldots \ldots \ldots \ldots \ldots \ldots . \ldots . \ldots \ldots$

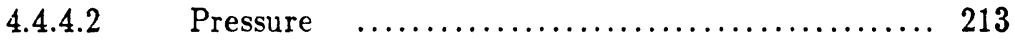

4.4.4.3 Particle Size $\ldots \ldots \ldots \ldots \ldots \ldots \ldots \ldots \ldots \ldots \ldots . \ldots \ldots$

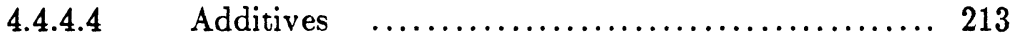

4.4.4.5 Pyrolysis Medium $\quad \ldots \ldots \ldots \ldots \ldots \ldots \ldots \ldots \ldots . \ldots 213$

4.4.5 Summary and Critique $\quad \ldots \ldots \ldots \ldots \ldots \ldots \ldots \ldots \ldots \ldots \ldots \ldots \ldots \ldots \ldots \ldots \ldots$

4.5 Pyrolysis of Lignocellulosic Materials $\ldots \ldots \ldots \ldots \ldots \ldots \ldots \ldots \ldots \ldots \ldots \ldots$

4.5.1 Low Temperature Phenomena $\ldots \ldots \ldots \ldots \ldots \ldots \ldots \ldots . \ldots . \ldots . \ldots$

4.5.2 Moderate Temperature Phenomena $\ldots \ldots \ldots \ldots \ldots \ldots \ldots \ldots . \ldots 216$

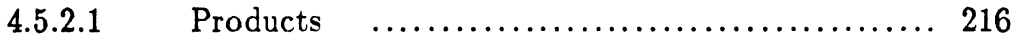

4.5.2.2 Mechanisms and Kinetics $\ldots \ldots \ldots \ldots \ldots \ldots . \ldots 217$

4.5.3 High Temperature Phenomena $\ldots \ldots \ldots \ldots \ldots \ldots \ldots \ldots \ldots . \ldots . \ldots . \ldots 21$

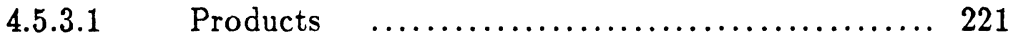

4.5.3.2 Mechanisms and Kinetics $\ldots . \ldots \ldots \ldots \ldots \ldots . \ldots 222$

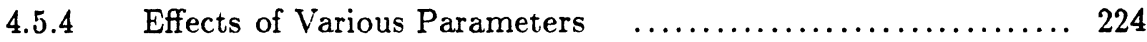

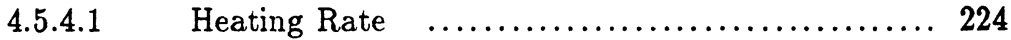

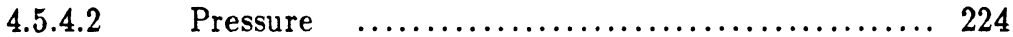

4.5.4.3 Particle Size $\ldots \ldots \ldots \ldots \ldots \ldots \ldots \ldots \ldots \ldots . \ldots . \ldots . \ldots . \ldots 224$

4.5.4.4 Gaseous Environment $\ldots \ldots \ldots \ldots \ldots \ldots \ldots \ldots . \ldots 224$

4.5.4.5 Mineral Matter and Additives $\ldots . \ldots \ldots \ldots . \ldots . . .224$

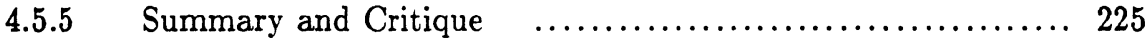




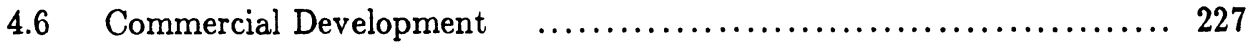

4.6.1 Generic Technologies $\ldots \ldots \ldots \ldots \ldots \ldots \ldots \ldots \ldots \ldots \ldots . \ldots \ldots \ldots \ldots$

4.6.2 Generic Economics ............................. 229

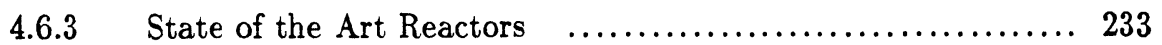

4.6.3.1 Class I Reactors $\ldots \ldots \ldots \ldots \ldots \ldots \ldots \ldots \ldots \ldots . \ldots . \ldots . \ldots 234$

4.6.3.2 Class II Reactors $\ldots \ldots \ldots \ldots \ldots \ldots \ldots \ldots \ldots . \ldots . \ldots 235$

4.6.3.3 Class III Reactors $\ldots \ldots \ldots \ldots \ldots \ldots \ldots \ldots \ldots . \ldots 236$

4.6.3.4 Summary and Critique $\ldots \ldots \ldots \ldots \ldots \ldots \ldots . \ldots 236$

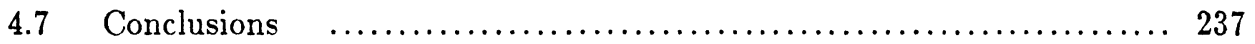

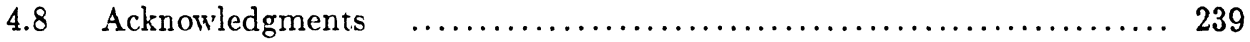

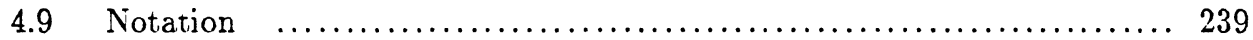

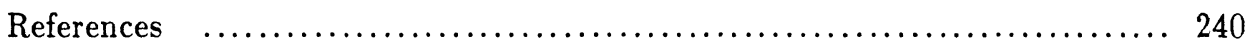

CHAPTER 5 Thermal Comfort and Passive Design

\section{S. V. Szokolay}

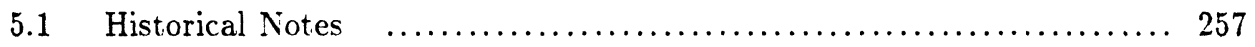

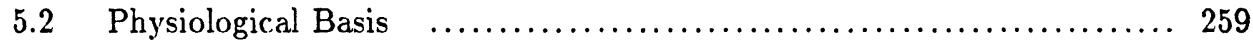

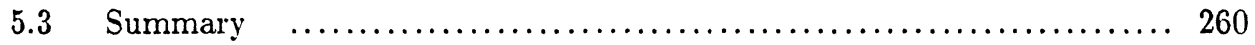

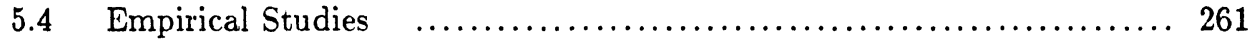

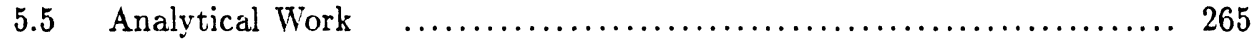

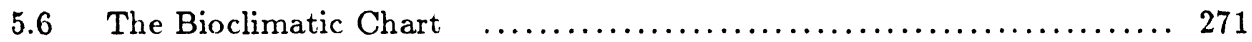

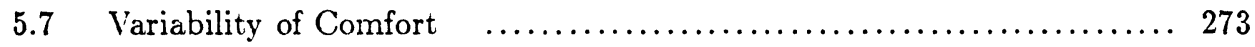

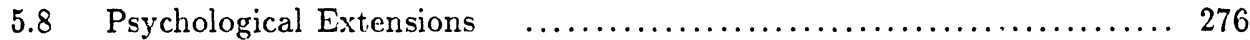

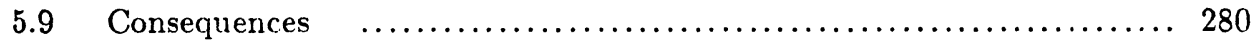

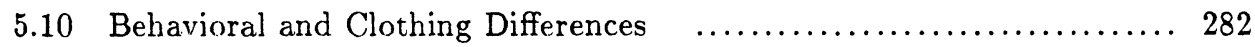

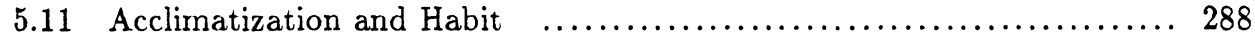

5.12 Passive Heating Systems and Comfort $\quad$........................ 290

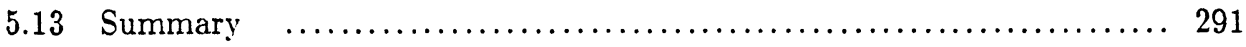

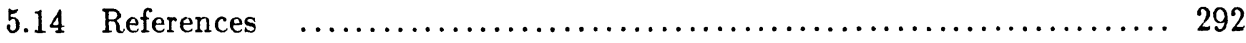


CHAPTER 6 Earth Contact Buildings: Applications, Thermal Analysis and Energy Benefits

John C. Carmody, George D. Meixel, Kenneth B. Labs and Lester S. Shen

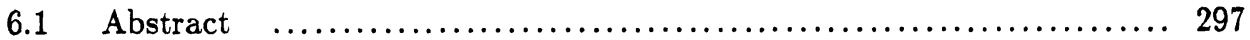

6.2 Earth Contact Structures and Their Applicability $\ldots \ldots \ldots \ldots \ldots \ldots . .297$

6.3 The General Advantages of Earth Contact Structures ............ 298

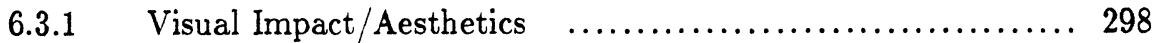

6.3.2 Preservation of Surface Open Space $\ldots \ldots \ldots \ldots \ldots \ldots \ldots \ldots \ldots . \ldots \ldots$

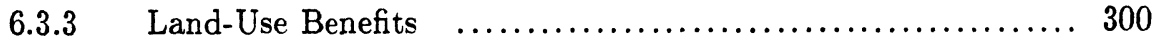

6.3.4 Environmental Benefits .............................. 300

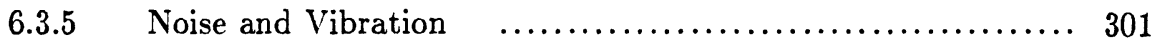

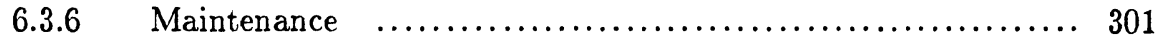

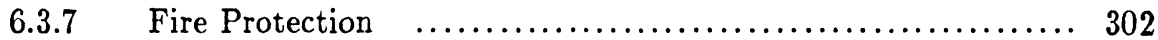

6.3.8 Protection from Earthquakes $\quad \ldots \ldots \ldots \ldots \ldots \ldots \ldots \ldots \ldots \ldots \ldots \ldots \ldots$

6.3.9 Suitability for Civil Defense $\quad \ldots \ldots \ldots \ldots \ldots \ldots \ldots \ldots \ldots \ldots \ldots \ldots \ldots$

6.3.10 Storm and Tornado Protection $\quad \ldots \ldots \ldots \ldots \ldots \ldots \ldots \ldots . \ldots . \ldots 2$

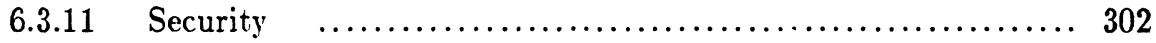

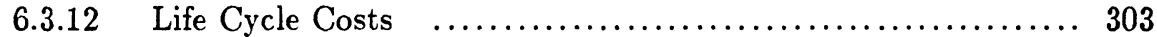

6.4 Potential Benefits Related to Energy Conservation $\ldots \ldots \ldots \ldots \ldots \ldots . \ldots 303$

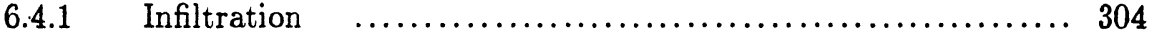

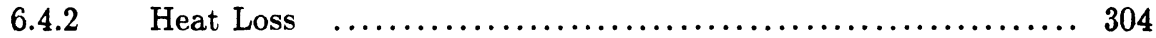

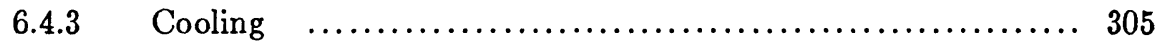

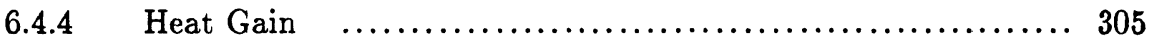

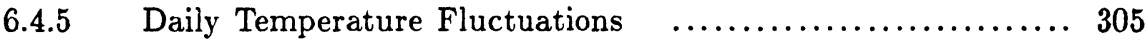

6.4.6 Seasonal Temperature Lag in Ground $\ldots \ldots \ldots \ldots \ldots \ldots \ldots \ldots . \ldots . \ldots 305$

6.5 Potential Limitations Related to Energy Conservation $\ldots \ldots \ldots \ldots \ldots . . .306$

6.5.1 Structural and Economic Limitations $\ldots \ldots \ldots \ldots \ldots \ldots \ldots \ldots \ldots$

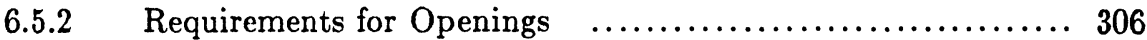

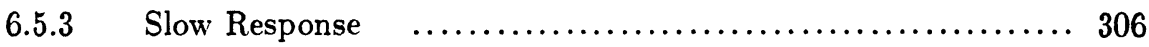

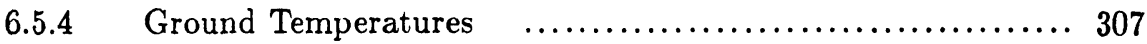

6.5.5 Drawbacks of Seasonal Time Lag in Temperatures $\ldots . . . . .307$ 
6.5.6 Heating/Cooling Compromises $\ldots \ldots \ldots \ldots \ldots \ldots \ldots \ldots \ldots \ldots . \ldots 308$

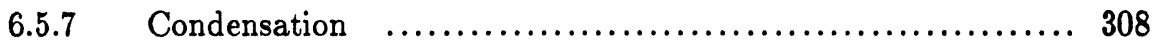

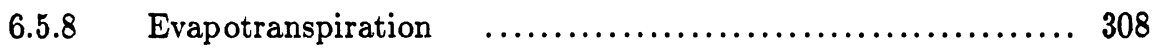

6.5.9 Indoor Air Quality $\ldots \ldots \ldots \ldots \ldots \ldots \ldots \ldots \ldots \ldots \ldots \ldots \ldots \ldots \ldots \ldots$

6.6 Application of Earth Contact Systems $\quad \ldots \ldots \ldots \ldots \ldots \ldots \ldots \ldots \ldots \ldots \ldots$

6.6.1 Residential Structures $\quad \ldots \ldots \ldots \ldots \ldots \ldots \ldots \ldots \ldots \ldots \ldots \ldots \ldots \ldots . \ldots \ldots$

6.6.2 Nonresidential Structures $\quad$............................ 309

6.6.3 Clusters of Buildings Employing Earth Contact ........... 311

6.6.4 Improved Exploitation of Earth Contact Potential ........ 311

6.7 Thermal Analysis of Earth Contact Buildings $\ldots \ldots \ldots \ldots \ldots \ldots \ldots . \ldots . \ldots . \ldots 11$

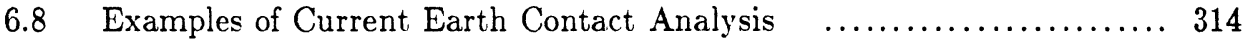

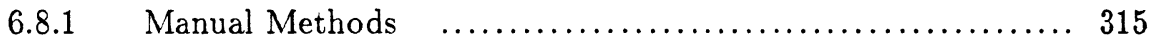

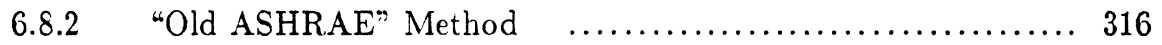

6.8.3 Method of Elliot and Baker $\quad \ldots \ldots \ldots \ldots \ldots \ldots \ldots \ldots \ldots \ldots \ldots \ldots \ldots \ldots$

6.8.4 Method of Boileau and Latta $\ldots \ldots \ldots \ldots \ldots \ldots \ldots \ldots \ldots \ldots \ldots \ldots \ldots$

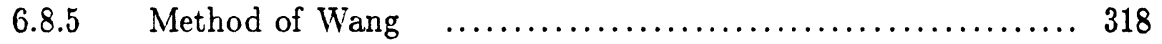

6.8.6 Method of Mitalas $\quad$................................. 319

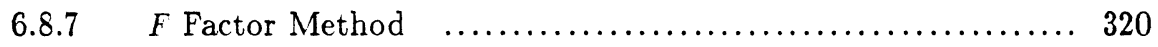

6.9 Investigations Using Computer Techniques $\quad \ldots \ldots \ldots \ldots \ldots \ldots \ldots \ldots \ldots . \ldots . \ldots 323$

6.10 Example of a Detailed Computer Analysis ...................... 324

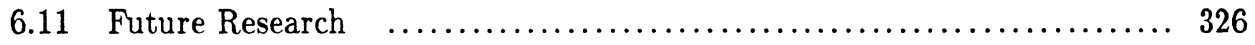

6.11.1 Comprehensive, Integrated Energy Analysis f........... 326

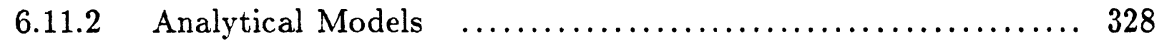

6.11.3 Measurements of Earth Contact Heat Transfer $\quad \ldots \ldots \ldots \ldots . . .330$

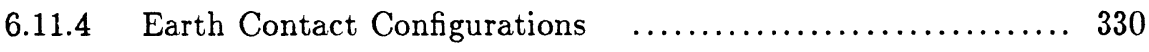

6.12 Energy Performance Analysis for Components of Small Earth Contact

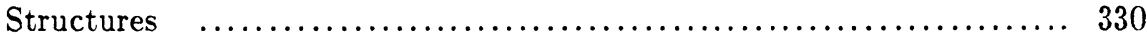

6.13 General Description of Parametric Studies .................... 331

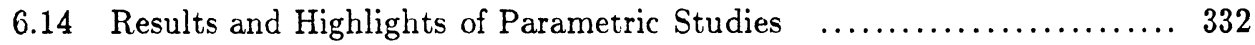

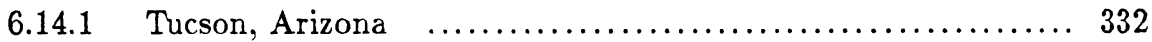

6.14.1.1 Above Grade Cases $\quad$....................... 332

6.14.1.2 Fully Bermed Case $\ldots \ldots \ldots \ldots \ldots \ldots \ldots \ldots \ldots . .333$ 
6.14.1.3 Earth Covered Case $\quad$....................... 333

6.14.1.4 Ground Surface Modifications $\ldots . \ldots \ldots \ldots \ldots . . . .333$

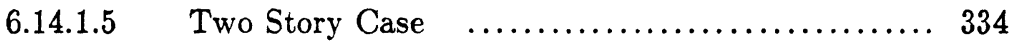

6.14.1.6 Monthly Distribution $\quad \ldots \ldots \ldots \ldots \ldots \ldots \ldots \ldots . \ldots 334$

6.14.2 Columbus, Ohio ................................ 337

6.14.2.1 Above Grade Cases $\quad$...................... 337

6.14.2.2 Fully Bermed Case $\ldots \ldots \ldots \ldots \ldots \ldots \ldots \ldots \ldots . \ldots . \ldots 337$

6.14.2.3 Earth Covered Case with a Typical Wall Insula-

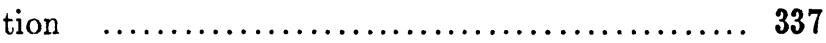

6.14.2.4 Earth Covered Case with Extended Roof Insulation $\ldots \ldots \ldots \ldots \ldots \ldots \ldots \ldots \ldots \ldots \ldots \ldots \ldots \ldots \ldots \ldots \ldots, 338$

6.14.2.5 Two Story Earth Covered Case $\quad \ldots . \ldots \ldots \ldots \ldots . . . .6339$

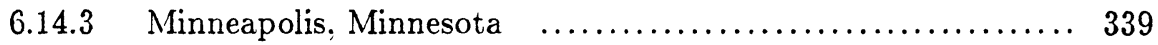

6.14.3.1 Above Grade Cases $\quad$...................... 339

6.14.3.2 Fully Bermed Case $\ldots \ldots \ldots \ldots \ldots \ldots \ldots \ldots \ldots . \ldots . \ldots . \ldots 340$

6.14.3.3 Earth Covered Case $\quad \ldots \ldots \ldots \ldots \ldots \ldots \ldots \ldots . \ldots . \ldots . \ldots 1$

6.14.3.4 Earth Covered Case with Floor Insulation $\quad \ldots . . .341$

6.14.3.5 Earth Covered Case with Extended Roof Insulation ................................... 341

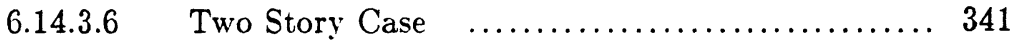

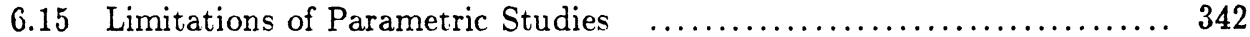

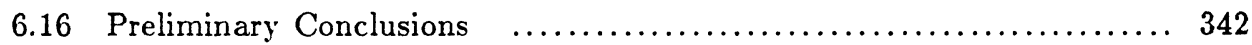

6.16.1 Building Configuration Considerations $\ldots \ldots \ldots \ldots \ldots \ldots \ldots . \ldots \ldots$

6.16.2 Interior Surface Considerations $\ldots \ldots \ldots \ldots \ldots \ldots \ldots \ldots \ldots, 343$

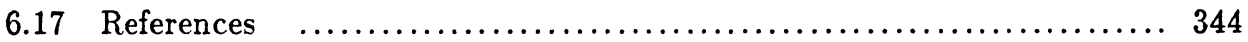

\section{CHAPTER 7 Testing Solar Collectors}

James E. Hill, Byard D. Wood and Kent A. Reed

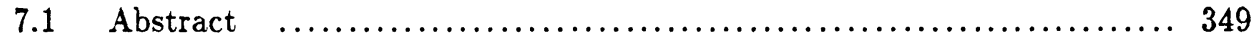

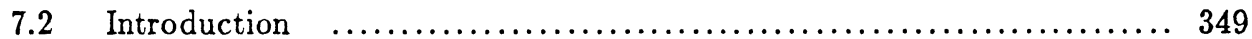

7.3 Basic Equations Governing the Thermal Performance of Solar Collectors 


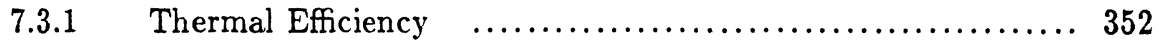

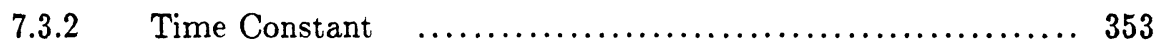

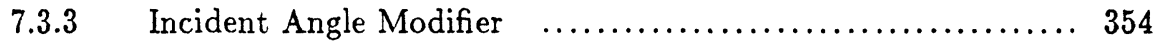

7.4 Testing Solar Collectors under Clear-Sky, Full-Irradiance Conditions 355

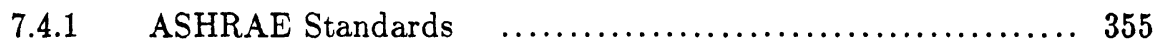

7.4.1.1 Time Constant Test $\quad \ldots \ldots \ldots \ldots \ldots \ldots \ldots \ldots \ldots . \ldots . \ldots 35$

7.4.1.2 Thermal Efficiency Test $\ldots \ldots \ldots \ldots \ldots \ldots \ldots . \ldots 360$

7.4.1.3 Incident Angle Modifier Test $\ldots \ldots \ldots \ldots \ldots \ldots . \ldots 362$

7.4.1.4 Instrumentation .......................... 364

7.4.2 Shortcomings of the Assumed Collector Model ........... 369

7.4.3 Comparability of Results from Outdoor Tests $\ldots \ldots \ldots \ldots . . . .373$

7.4.4 Testing Concentrating Collectors $\ldots \ldots \ldots \ldots \ldots \ldots \ldots \ldots \ldots$

7.5 Testing Solar Collectors under Zero-Irradiance Conditions $\ldots \ldots \ldots \ldots . .384$

7.6 Considerations in Testing Air Collectors $\ldots \ldots \ldots \ldots \ldots \ldots \ldots \ldots \ldots \ldots . \ldots \ldots$

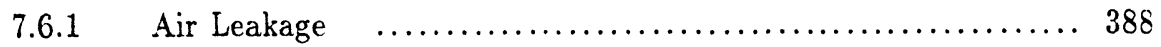

7.6.2 Predicting Collector Array Performance from Tests on Mod-

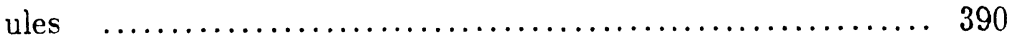

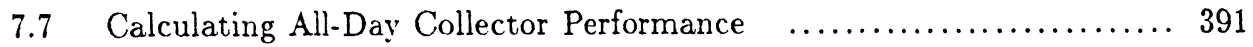

7.7.1 Calculation Including Diffuse Solar Irradiance $\ldots \ldots \ldots \ldots \ldots . \ldots 391$

7.7.2 SRCC Rating Calculation Methods $\quad$.................... 393

7.7.3 Effects of Diffuse Irradiance on Calculations .............. 394

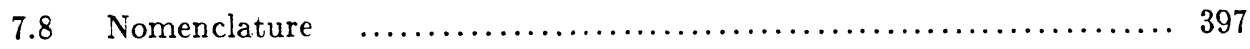

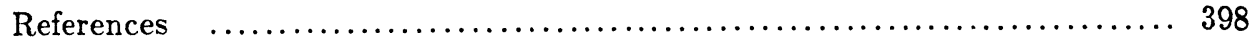

\section{CHAPTER 8 Concentrating Solar Collectors}

\section{A. Rabl}

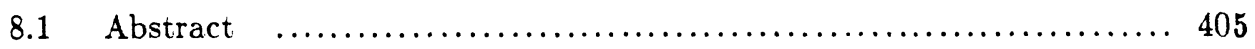

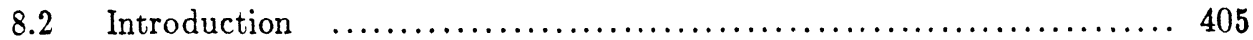

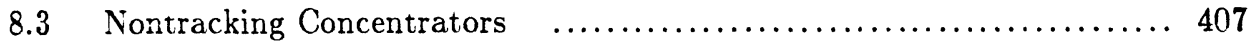

8.3.1 Compound Parabolic Concentrators $\quad$................... 408

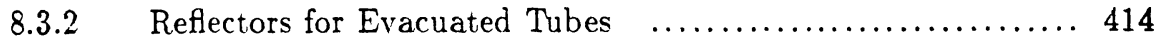

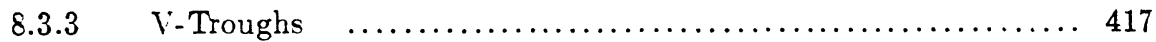


8.3.4 Side Reflectors $\quad \ldots \ldots \ldots \ldots \ldots \ldots \ldots \ldots \ldots \ldots \ldots \ldots \ldots \ldots \ldots \ldots \ldots \ldots \ldots, 418$

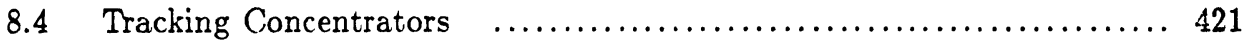

8.4.1 Image Spread Due to Finite Width of the Sun and Optical Errors

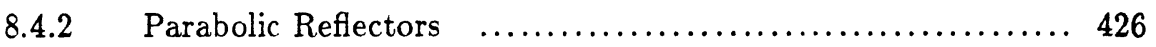

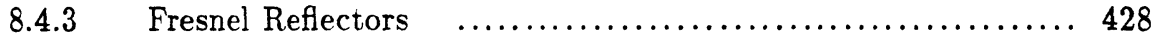

8.4.4 Fresnel Lenses $\ldots \ldots \ldots \ldots \ldots \ldots \ldots \ldots \ldots \ldots \ldots \ldots \ldots \ldots . \ldots \ldots \ldots$

8.4.5 Fixed Reflectors with Tracking Receivers $\quad \ldots \ldots \ldots \ldots \ldots \ldots . \ldots 437$

8.4.5.1 Spherical Reflectors $\ldots \ldots \ldots \ldots \ldots \ldots \ldots \ldots \ldots . \ldots 437$

8.4.5.2 Circular Cylindrical Reflector with Tracking Re-

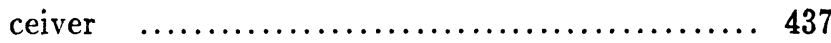

8.4.5.3 Reflector Slats on Circular Cylindrical Mount .. 439

8.4.6 Concentrator Configurations for Low Cost Manufacture ... 440

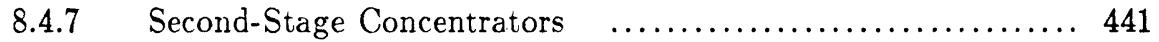

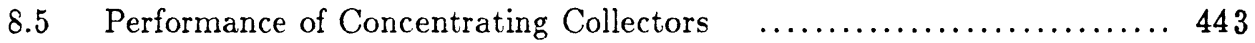

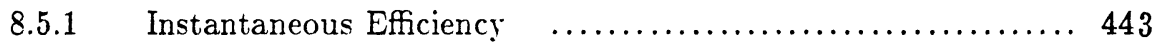

8.5.2 Long-Term Average Performance $\ldots \ldots \ldots \ldots \ldots \ldots \ldots \ldots . \ldots . \ldots 48$

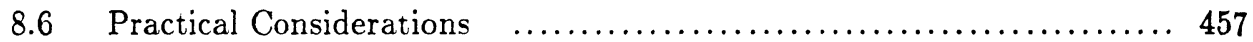

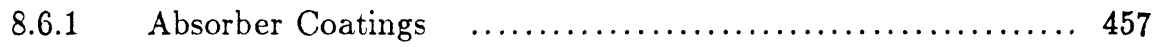

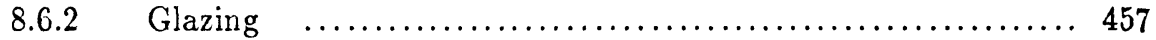

8.6.3 Reflector Materials $\ldots \ldots \ldots \ldots \ldots \ldots \ldots \ldots \ldots \ldots \ldots \ldots, 461$

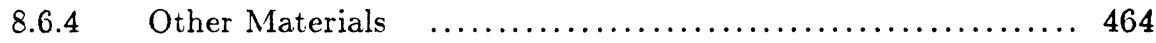

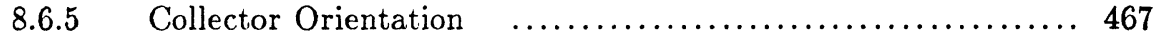

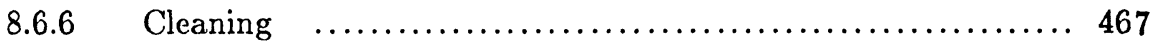

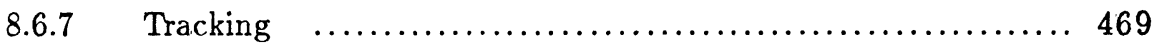

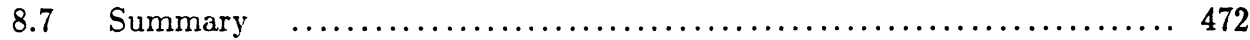

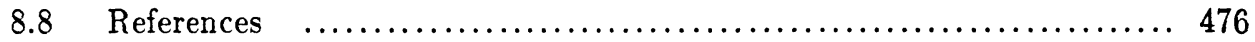

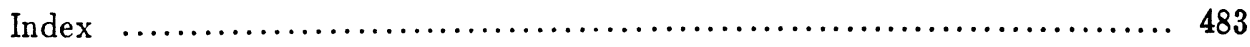




\section{ABOUT THE AUTHORS}

\section{CHAPTER 1}

Ronald Stewart is a Research Professor at the Atmospheric Sciences Research Center, State University of New York at Albany. He directed: (1) The U.S. DOE sponsored Solar Energy Meteorological Research and Training Site: Region 2, (2) the operation of the photovoltaic communication system at the 1980 Winter Olympics, (3) the statewide energy audit program (8500 energy audits) including ten colleges, and (4) the analysis of an alumini house-conference center which had an active solar heating system.

In addition Mr. Stewart has published over 100 articles, reports, etc. and directed over $\$ 4$ million in research projects. He is a member of ISES, ASES, and the Royal Meteorological Society.

Daniel Spencer is a Program Coordinator at the University of Wisconsin. He received his doctorate in atmospheric sciences from the State University of New York at Albany in 1983. His dissertation title was "Clear Atmospheric Effects on Insolation Illustrated by Broadband Radiometer Measurements." Spencer has published articles in several journals and presented professional papers in Europe, Australia, and the U.S. Previously he developed the computerized quality control programming for the U.S. DOE supported Solar Energy Meteorological Research and Training Site: Region 2, and studied lightning flash frequency based upon satellite photography.

Richard Perez is a Research Associate at the Atmospheric Sciences Research Center, State University of New York at Albany. He is currently Project Director of a daylight availability resource assessment program. This project involves the measurement of over 20 radiation/daylight parameters, as well as the study of their interrelationship.

As a participant to the U.S. DOE funded Solar Energy Meteorological Research and Training Sites program, he has done extensive radiation modeling work; the anisotropic diffuse model he developed is now assessed by SERI and Sandia National Laboratories for potential large scale utilization. This work also involved collector geometry optimization for photovoltaic applications. 


\section{CHAPTER 2}

Richard E. Speece is Betz Chair Professor of Environmental Engineering at Drexel University. He received his Bacheior's, Master's. amd $\mathrm{PhD}$. from Penn Collge, Yale University and MIT respectively.

He received the Eddy Medal from the Water Polution Control Federation and the Croes Medal from the American Society of Civil Engineers. He received the Distinguished Faculty Award, the Distinguished Lecturer Award and the Engineering Science Award from the Association of Environmental Engineering Professors. He is listed in Who's Who in Engineering, America and the World.

\section{CHAPTER 3}

Thomas B. Reed is currently principal scientist in the Thermochemical and Electrochemical research branch at the Solar Energy Research Institute (SERI) in Golden, Colorado. He obtained his $\mathrm{PhD}$. in physical chemistry from the University of Minnesota in 1952 in the field of crystallography. He worked for the Linde division of Union Carbide from then until 1959 in the field of high temperature processing and chemicals. In 1960 he went to the MIT Lincoln Laboratory and worked in the field of materials science and energy until 1977, when he went to SERI to continue his work in the field of renewable energy, espcially alcohols from biomass. He has published over 200 papers in the fields of high temperature processes, materials science, renewable energy and thermodynamics. In 1965 he spent a year at Oxford University as Senior Research Fellow. He was given an IR 100 award in 1983 for invention of the high pressure oxygen biomass gasifier.

\section{CHAPTER 4}

Michael Jerry Antal, Jr. graduated Summa Cum Laude with Highest Distinction in Physics and High Distinction in Mathematics from Dartmouth College in 1969. He earned an MS in Applied Physics in 1970, and a PhD. in Applied Mathematics in 1973, both from Harvard University. Afterwards, Dr. Antal spent two years as a theorist with the Thermonuclear Weapons Physics Group of the Los Alamos Scientific Laboratory, and six years with Princeton University. At Princeton he was a member of the faculty of the Mechanical and Aerospace Engineering Department and Director of the Renewable Resources Research Laboratory $\left(R^{3} L\right)$. In 1981 Dr. Antal was invited to assume the newly endowed Coral Industries Distinguished Professor of Renewable Energy Resources Chair with the University of Hawaii, where he is now engaged in research on the pyrolysis of biomass materials, and high temperature solar thermal energy utilization.

\section{CHAPTER 5}

Steven V. Szokolay is Director of the Architectural Science Unit at the University of Queensland, Brisbane, Australia. He was born and brought up in Hungary, qualified as an architect in Sydney, and has an Master's of Architecture degree of Liverpool (U.K.) and a PhD. of Queensland university. For some ten years he was 
teaching at Liverpool and London. He designed the first solar heating system in the U.K. for a house at Milton Keynes new town (1973). He is co-author (with Koenigsberger) of a book on climatic design, the author of Solar Energy and Building (1975), World Solar Architecture (1980), Environmental Science Handbook for Architects (1980), ANZ Solar Home Book (1981), Climatic Data and its Use in Design (1982), and of innumerable papers, articles, and other contributions. Currently his work is aimed at design tool development, in order to integrate solar design with the everyday practice of architeccture.

\section{CHAPTER 6}

John Carmody is an architect and is currently the Associate Director of the Underground Space Center at the University of Minnesota. After receiving a Bachelor of Architecture degree from the University of Minnesota, he specialized in the design and construction of housing while in the Peace Corps and later in the United States. Mr. Carmody has been the co-author of five books on energyefficient and underground building design. At the Underground Space Center, he has worked on a wide variety of research projects including underground space planning and development, determining the energy performance of housing, and developing design guidelines for energy-efficient buildings.

George Meixel, Jr. received a Bachelor of Science degree in Engineering Physics in 1967, and a PhD. in Aerospace Engineering and Plasma Physics in 1973 from Cornell University. Dr. Meixel's research experience includes hypersonic aerodynamics, controlled fusion, coal gasification, computer graphics, and energy conservation in buildings. As a research associate at the Underground Space Center at the University of Minnesota for six years, Dr. Meixel has specialized in computer-based theoretical studies and experimental measurements of the heat transfer characteristics of underground buildings. Recently, Dr. Meixel was the Principal Investigator for a multi-year research project on passive cooling through earth contact structures for the U.S. DOE.

Kenneth Labs received his Master's of Architecture degree from Washington University in 1975 , and presently maintains his own design and research practice in New Haven, Connecticut, where he is a Visiting Lecturer in Environmental Technology at the Yale School of Architecture. He is the author of over 30 publications dealing with earth coupled building design, including the award-winning report to the U.S. DOE, "Regional Analysis of Ground and Above Ground Climate." He is also co-author (with Donald Watson, FAIA), of Climatic Design: Energy Efficient Building Principles and Practices (McGraw-Hill, 1983).

Lester Shen received his Bachelor's degree in Chemistry at Haverford College in 1977, and a Master's of Science degree in Mechanical Engineering from the Georgia Institute of Technology. Currently at the Unversity of Minnesota, Mr. Shen is completing his $\mathrm{PhD}$. in mechanical engineering, studying coupled heat and moisture flow in soils. He is presently a researcher at the Underground Space Center. Since being at Minnesota, he has been involved in research dealing with the thermal performance of earth-sheltered construction, building load simulation programs, and weatherization of residential housing. 


\section{CHAPTER 7}

James E. Hill (PhD. Georgia Institute of Technology, 1968) is the Chief of the Building Equipment Division at the National Bureau of Standards. He is responsible for applied research and the development of testing and performance standards for building equipment. He was Assistant Professor of Mechanical Engineering at the University of Maryland for three years before joining NBS in 1972.

Dr. Hill is a member of ASHRAE, the American Society of Mechanical Engineers, the Society of Sigma Xi, and ISES. He has authored over 50 technical papers in the building research/solar energy areas.

Byard D. Wood is a Professor of Mechanical Engineering at Arizona State University, Tempe, Arizona. He received his Bachelor of Science and Master's of Science degrees from Utah State University and his $\mathrm{PhD}$. from the University of Minnesota. A registered professional engineer, he is an active member of several professional societies.

Dr. Wood has served on a number of national and international committees concerned with the development and evaluation of test methods for solar collectors and solar systems. He is chairman of the revision committee for ASHRAE Standard 93-77, chairman of the Technical Compliance Committee for Solar Rating and Certification Corporation, member of the U.S. delegation to International Standards Organization TC 180 Solar Energy. He is an Associate Editor of the Journal of Solar Energy Engineering.

Kent A. Reed (PhD. The University of Chicago, 1976) is the Leader of the Computer Integrated Construction Group at the National Bureau of Standards. $\mathrm{He}$ is responsible for planning and executing projects. For three and half years, Dr. Reed was the leader of the Solar Equipment Group at NBS. Before joining NBS, Dr. Reed was staff scientist in the Solar Energy Group at Argonne National Laboratory for four years.

Dr. Reed is a member of the American Society for Testing and Materials (ASTM), ISES, ASES, the American Physical Society, and the American Association for Artificial Intelligence.

\section{CHAPTER 8}

Ari Rabl is research scientist at the Center for Energy and Environmental Studies of Princeton University, where he has been since 1980. A recipient of a Fulbright grant, he obtained his $\mathrm{PhD}$. in theoretical high energy physics from the University of California at Berkeley in 1969. He continued in this field during postdoctoral appointments at the Weizmann Institute and Ohio State University until 1973 where he began work in solar energy at the Argonne National Laboratory, the University of Chicago, and the Solar Energy Research Institute.

He has published over fifty journal articles on high energy physics, applied optics, heat transfer, and solar energy; and he holds five patents on solar collectors. He is author of Active Solar Collectors and their Applications (Oxford University Press, 1985). He has lectured and consulted extensively, both in the U.S. and abroad; and at Princeton he has taught courses on energy and environment and on solar energy. 